\begin{tabular}{|c|l|}
\hline Title & Heterogeneity-induced defect bifurcation and pul se dynamics for a three component reaction-diffusion system. \\
\hline Author(s) & Yuan, Xiaohui; Teramoto, Takashi; Nishiura, Y asumasa \\
\hline Citation & $\begin{array}{l}\text { Physical Review E, 75(3 Pt 2), 036220 } \\
\text { https://doi.org/10.1103/PhysRevE.75.036220 }\end{array}$ \\
\hline Issue Date & 2007-03-27 \\
\hline Doc URL & http://hdl.handle.net/2115/22083 \\
\hline Rights & Copyright $\odot 2007$ American Physical Society \\
\hline Type & article \\
\hline File Information & PRE75-3.pdf \\
\hline
\end{tabular}

Instructions for use 


\title{
Heterogeneity-induced defect bifurcation and pulse dynamics for a three-component reaction-diffusion system
}

\author{
Xiaohui Yuan \\ Department of Mathematics and Research Institute for Electronic Science, Hokkaido University, Sapporo 060-0813, Japan \\ Takashi Teramoto \\ Department of Photonics Material Science, Faculty of Photonics Science, Chitose Institute of Science and Technology, \\ Chitose 066-8655 Japan \\ Yasumasa Nishiura* \\ Research Institute for Electronic Science, Hokkaido University, Sapporo 060-0813, Japan
}

(Received 24 November 2006; published 27 March 2007)

\begin{abstract}
We consider the dynamics when traveling pulses encounter heterogeneities in a three-component reaction diffusion system of one-activator-two-inhibitor type, which typically arises as a qualitative model of a gasdischarge system. We focused on the case where one of the kinetic coefficients changes similar to a smoothed step function, which is basic for more general heterogeneity as in periodic or random media. Since the heterogeneity is introduced to the kinetic part in an additive way, it causes the system to produce various types of localized structures smoothing the jump heterogeneity called the defects at the jump point, which makes a sharp contrast with the multiplicative heterogeneous case for the Gray-Scott model. The main issue is to study the collision dynamics between traveling pulses and defects, and show that their global bifurcation structure plays a key role in clarifying the underlying mechanism. Five outputs are observed after collisions including annihilation, rebound, and pinning. Unstable steady states are identified as separators between two different dynamic regimes: penetration and rebound, the role of which is very close to that of scattors arising in collision process. An organizing center producing the traveling pulses, defects, and scattors via unfolding with respect to the parameters is also presented.
\end{abstract}

DOI: 10.1103/PhysRevE.75.036220

PACS number(s): 82.40.Bj, 68.35.Fx, 82.20.Wt, 89.75.Kd

\section{INTRODUCTION}

Spatially localized patterns such as pulses and spots are fundamental objects arising in many dissipative systems, see, e.g., Refs. [1-7]. One of the recent remarkable discoveries is that there is a class of localized patterns which displays a variety of dynamics such as elastic ball-like behaviors upon collision, self-replication, self-destruction, and spatiotemporal chaos [8-22]. This makes a sharp contrast with wellknown classical excitable waves as in the FitzHugh-Nagumo (FHN) equations in which annihilation is typically observed upon collision [23].

One of the origins of such rich behaviors is that the pulses have potential instabilities that display a variety of dynamics when parameters are tuned appropriately. For instance, saddle-node structure causes self-replication [24] or selfdestruction [25], and drift bifurcation is responsible for the onset of traveling motion. Moreover a singularity of codim 2 type, i.e., a system has a parameter where multiple bifurcations simultaneously occur, is known to be responsible for the variety of outputs for scattering process $[14,15,26]$.

Here we explore numerically the pulse dynamics when the media is changed from homogeneous to heterogeneous, especially focused on the case where one of the kinetic coefficients changes similar to a smoothed step function as in Eq. (2). Although this heterogeneity of jump type looks spe-

\footnotetext{
*Electronic address: nishiura@aurora.es.hokudai.ac.jp
}

cial, all the key features of the subsequent results including the defect structure, diversity of the outputs, and structure of the organizing center remain the same for the class of steep heterogeneity of jump type [i.e., large $\gamma$ for Eq. (2)] up to step function with discontinuity. Note that the pulses treated here are asymptotically stable in homogeneous media, however we assume that the associated parameter values are located close to the singularities such as drift, saddle-node, and Hopf bifurcations. One of the consequences of this assumption is the enhancement of sensitivity to the perturbations: for instance, if there is a traveling pulse close to the drift bifurcation, i.e., the onset of transition from standing pulse to traveling one. its profile is almost symmetric and easy to deform from the right-going pulse to the left-going one by the external perturbations, which actually occurs when two slowly traveling pulses collide, interact weakly, and bounce off [10]. A similar thing occurs when the pulse encounters the heterogeneity as will be discussed in Sec. III, and much more exotic dynamics are created than the well-studied case for the FHN equations [27-29] and the front case [30-34].

To be specific, we employ the three-component reaction diffusion system of Eq. (1) as a representative model and consider the case in which one of the kinetic parameters $k_{1}$ changes abruptly around a point. The model system (1) was derived phenomenologically from gas discharge experiments $[1,35]$ in which a variety of spot dynamics including splitting, clustering, and other complex dynamics are observed. Our previous paper [36] discusses a similar problem as above for the Gray-Scott (GS) model and showed various 
pulse dynamics including rebound and splitting depending on the height and slope of the heterogeneity for the removal rate $k$. A big difference between the GS model and the threecomponent model (1) is the dependency of the background state on the kinetic parameter. Here the background state is the rest state for a monostable system after introducing heterogeneity. For the GS model, background state $(1,0)$ is independent of $k$, therefore it remains a common constant state before and after the jump point. On the other hand, since heterogeneity is introduced in an additive way for Eq. (1), there are no such common constant states for our system. Therefore it is not a priori clear that there exists a smooth stable background state connecting two different constant states on the whole region called the defect; in fact, it is not always the case (see Sec. VII). Such defects, if exist, turn out to be coexistent in general for a fixed set of parameters. We particularly focus on the case that the traveling pulse collides with the small defect introduced in Sec. IV. The defect branch consisting of its global continuation with respect to the height of the jump and $k_{1}$ plays a key role to understand the output when the pulse hits the heterogeneity.

When the heterogeneity is spatially localized similar to a Gaussian distribution, heterogeneity-induced stationary and oscillatory patterns were studied in detail by Refs. [34,37] for the Fitzhugh-Nagumo system and integrodifferential equations. They found several interesting heterogeneityinduced bifurcations including a pulse generator, however they did not consider how the traveling pulses interact with those structures. The main theme of this paper is first to study existence and stability of defects, and secondly to investigate the dynamics of traveling pulses of Eq. (1) that encounter with the defects created by the heterogeneities of jump type. There are five different outputs depending on the parameters: penetration, rebound, annihilation, oscillation, and steady state. A remarkable thing is that there are unstable steady states which act as separators near the boundary of penetration-rebound regions. The role of them is exactly the same as scattors for the case of head-on collisions of two traveling pulses discussed by Refs. [14,15]. Moreover we show that those unstable patterns can be obtained as a global continuation of defects with respect to the jump size.

The paper is organized as follows. In Sec. II, we introduce our model system. In Sec. III, existence and stability properties of standing and traveling pulses are studied for the homogeneous case. We introduce the heterogeneity in Sec. IV and show the existence of heterogeneity-induced defects. Phase diagram of collision dynamics between traveling pulse and small defect is presented. In Sec. V, we study how collision dynamics is controlled by unstable defects with the aid of careful numerics around the transition points of outputs. In Sec. VI, an organizing center for the dynamics in heterogeneous media is presented, which produces all the important objects including standing and traveling pulses, and defects via unfolding. The organizing center is, loosely speaking, a merging point of saddle-node, Hopf, and drift bifurcations located on the defect branch. We conclude the discussions in Sec. VII and present several open questions including a pulse generator induced by heterogeneity.

\section{MODEL SYSTEM}

In order to investigate the dynamics of traveling pulses in heterogeneous media, we employ the following threecomponent reaction diffusion system (1), which was proposed as a qualitative model of gas discharge phenomena [3]:

$$
\begin{gathered}
u_{t}=D_{u} \Delta u+k_{2} u-u^{3}-k_{3} v-k_{4} w+k_{1}, \\
\tau v_{t}=D_{v} \Delta v+u-v, \\
w_{t}=D_{w} \Delta w+u-w,
\end{gathered}
$$

where $\Delta$ is the Laplacian, $u=u(t, x), v=v(t, x)$, and $w=w(t, x)$ depend on time $t$ and $x \in R^{1}, k_{1}, k_{2}, k_{3}$, and $k_{4}$ are kinetic parameters, $\tau$ and the diffusion coefficients $D_{u}, D_{v}$, $D_{w}$ are positive constants. In view of the nonlinearity, Eq. (1) can be regarded as a generalization of the well-known Bonhoeffer-van der Pol kinetics to the three component system by adding the second inhibitor $w$. The third component $w$ is indispensable for the coexistence of multiple number of stable traveling spots in higher dimensional spaces. The system (1) is simple and a prototypical model for the study of interaction among moving particle patterns in dissipative systems. Note that there is a variant of Eq. (1) in Ref. [38] for the case where total change is conserved. We employ the following parameter values in the subsequent sections $k_{2}=2.0, \quad k_{3}=1.0, \quad k_{4}=8.5, \quad\left(D_{u}, D_{v}, D_{w}\right)=\left(0.9 \times 10^{-4}\right.$, $\left.1.0 \times 10^{-3}, 1.0 \times 10^{-2}\right)$ and only two parameters $\left(k_{1}, \tau\right)$ are varied. The kinetic ODE part of Eq. (1) has, in general, three critical points $U_{0}=\left(u_{0}, v_{0}, w_{0}\right)$ satisfying $u_{0}=v_{0}=w_{0}$. We assume in this paper that $k_{1}$ varies only in the interval $[-7.0,-6.0]$, which guarantees that the kinetic system has a unique stable critical point $U_{0}$, i.e., Eq. (1) is monostable. More precisely the bifurcation diagram of the kinetic system is obtained by using the AUTO software [39] as shown in Fig. 1, in which the gray-colored area is the region we are concerned with.

\section{HOMOGENEOUS MEDIA}

In order to study the pulse dynamics in heterogeneous media, we need to know the existence and stability properties of traveling pulses in homogeneous media. Traveling pulses typically emerge from standing ones via drift bifurcation, so that location of standing pulses in a parameter space and the detection of drift bifurcation are the basic things to do. Figure 2 shows the typical observable patterns of Eq. (1) in two-dimensional parameter space $\left(k_{1}, \tau\right)$ starting from a spatially localized initial condition. There are three different regimes: standing pulses (light gray), traveling pulses (dark gray), and ground states (black). The ground state means that the solution goes to the unique constant state $U_{0}$. Note that the ground state always exists as one of the stable solutions in this parameter range. The structure of steady state solutions is independent of $\tau$ except the stability properties, in fact the location of the saddle-node (SN) point (solid white line in Fig. 2 at $k_{1} \approx-6.80$ ) for the standing pulse branch does not depend on $\tau$. On the other hand, locations of the 


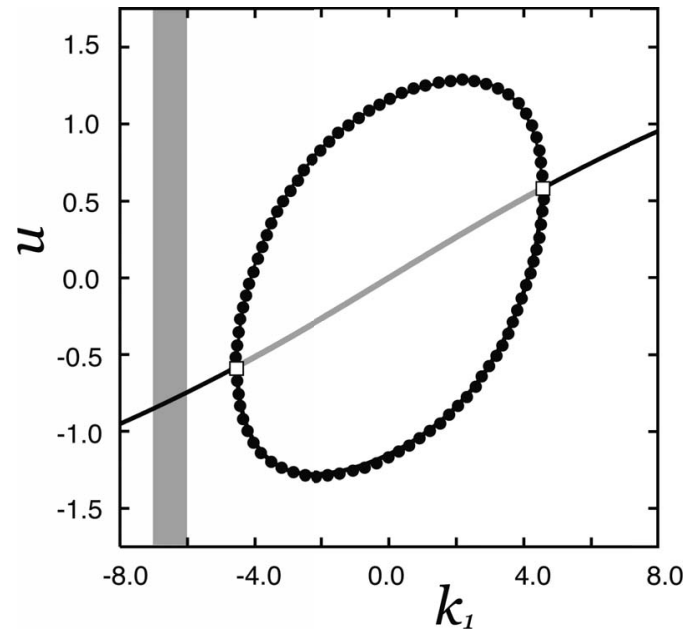

FIG. 1. Bifurcation diagram of the kinetic part of the model system (1): Solid and gray lines stand for the stable and unstable branch of the critical points. White squares show the location of the Hopf bifurcation points at $\left|k_{1}\right| \approx 4.5$. Filled circles stand for the maximum and minimum values of stable periodic motion. The gray strip indicates the range of $k_{1}$ parameter $[-7.0,-6.0]$ studied here, which is part of the monostable region.

Hopf and drift bifurcations on it depend on $\tau$, although the Hopf line (white broken line around $k_{1} \approx-6.73$ ) looks almost similar to a straight line. Stable standing pulse solutions exist in the right-low region of Fig. 2, but they are destabilized by the Hopf bifurcation as $k_{1}$ is decreased before reaching the saddle-node line. The boundary between standing and traveling regions coincides with a part of the drift bifurcation (white dotted) line for the standing pulse. In fact, stable traveling pulses emanate supercritically from the boundary line. As $k_{1}$ is decreased, the drift line intersects with the Hopf line

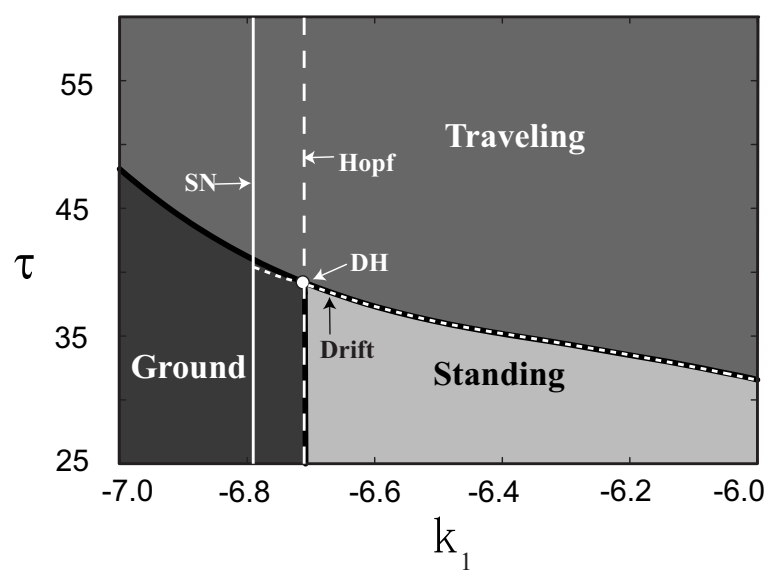

FIG. 2. Phase diagram in the $\left(k_{1}, \tau\right)$ plane for homogeneous system. The dark and light gray regions indicate stable traveling and standing pulses, respectively. The black region denotes the ground constant state, i.e., there are no pulses. Triple junction of these three regions is located around $(-6.73,39.3)$. The vertical broken (solid) white line stands for the Hopf (saddle-node) bifurcation of the standing pulse. The dotted white line stands for the drift bifurcation which intersects with the Hopf line at DH of codim 2 point. Note that the triple junction point coincides with the codim 2 point $\mathrm{DH}$.

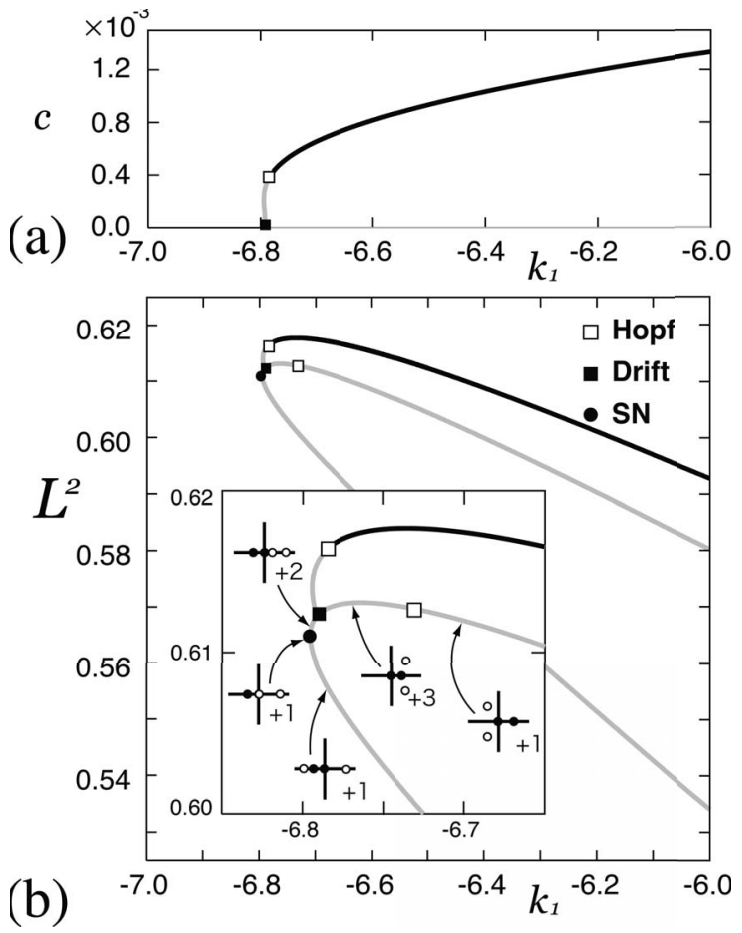

FIG. 3. (a) The propagating velocity of traveling pulse as a function of $k_{1}$ for $\tau=40.0$. Solid and gray lines indicate stable and unstable solutions, respectively. (b) Global bifurcation diagram for standing and traveling pulses in homogeneous media. The ordinate $L^{2}$ stands for the integral norm of square of $u$. The upper branch stands for the traveling pulses of (a) emanating from the standing pulse branch (gray line) via the drift bifurcation (filled square). Inset: saddle-node bifurcation (filled circle) occurs for the standing pulse branch at $k_{1} \approx-6.80$. The white and filled square represent the Hopf and drift bifurcation points at $k_{1} \approx-6.73$ and -6.79 , respectively. Schematic behaviors of eigenvalues for standing pulse are also depicted along the branch.

at $(-6.73,39.3)$ and can be extended up to the $\mathrm{SN}$ line. The intersection is a codim 2 point and denoted by DH. After crossing DH $(\tau>\mathrm{DH})$, the drift bifurcation becomes subcritical [see Fig. 3(a) for $\tau=40.0$ ] so that it is no more a part of the boundary of traveling pulse region. As shown in Fig. 3, traveling pulse recovers its stability via the Hopf bifurcation. This implies that the boundary of the existence of stable traveling pulses on the left side of DH is taken over by this Hopf recovery line. The DH point turns out to be a triplejunction of these three dynamic regimes. Note that the velocity of traveling pulses near DH is very small. Figure $3(\mathrm{~b})$ shows a global bifurcation diagram of standing and traveling pulses with respect to $k_{1}$ at $\tau=40.0$ slightly above the DH point. The Hopf (white square) and drift (filled square) bifurcation points on the standing pulse branch are located at $k_{1} \approx-6.73$ and -6.79 , respectively. The profile of the Hopf eigenfunction $\Phi_{1}$ at $k_{1} \approx-6.79$ is depicted in Fig. 4(b) and its eigenvalue is computed as $\lambda_{1}=0.080 \pm 0.012 i$. The Hopf eigenmode $\Phi_{1}$ suggests the up-down motion of the pulse. On the other hand, the drift eigenmode $\Psi_{2}$ implies the distortion of the symmetric standing pulse leading to left-right going pulse. The deformation vector $\Psi_{2}$ is obtained by solving the generalized eigenvalue problem. Schematic behaviors of ei- 
(a)

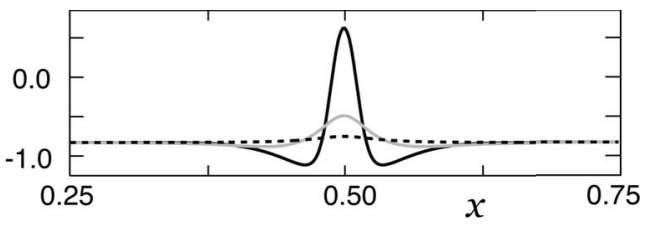

(b)
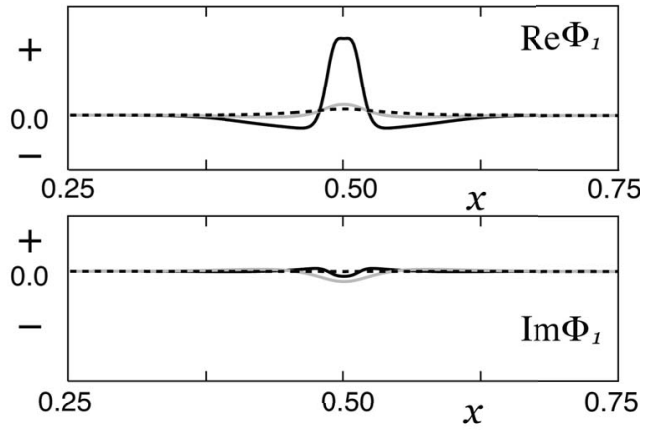

(c)

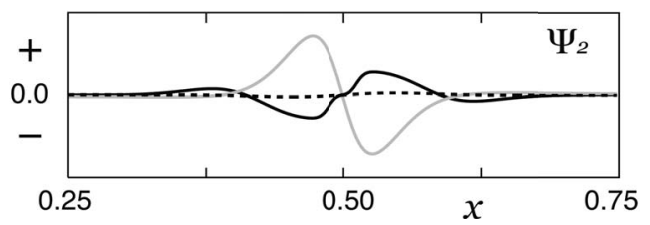

FIG. 4. (a) The profile of the unstable standing pulse at the drift point in Fig. 3 ( $k_{1} \approx-6.79$ denoted by the filled square). There are two unstable modes: one is Hopf and the other is drift one [see the inset of Fig. 3(b)]. The associate eigenfunctions $\Phi_{1}$ and $\Psi_{2}$ are depicted in (b) and (c), respectively. The solid, gray and dotted lines corresponds to the $u, v$, and $w$ component, respectively. The generalized eigenfunction $\Psi_{2}$ is obtained by solving $\mathcal{L} \Psi_{2}=-\Phi_{2}$ where $\Phi_{2}$ is the Goldstone mode, i.e., the spatial derivative of standing pulse (a). Schematic behaviors of eigenvalues for standing pulse are also depicted along the branch in the inset of Fig. 3(b).

genvalues for standing pulse are also depicted along the branch of Fig. 3(b). The white (filled) circle means the updown (left-right) eigenvalue in the complex plane. Note that there always exists a translation zero eigenvalue at the origin. A pair of complex eigenvalues falls into the real axis before the SN point and one of them is responsible for the occurrence of saddle-node bifurcation. The recovery mechanism of the traveling pulse branch due to the Hopf bifurcation [located at $k_{1} \approx-6.78$ in Fig. 3(b)] results from the unfolding of the codim 2 point $\mathrm{DH}$ for the standing pulse branch. More detailed discussions will be reported in Ref. [26].

\section{HETEROGENEOUS MEDIA}

We introduce a smooth heterogeneity to the parameter $k_{1}$ of Eq. (1) in the following way:

$$
k_{1}(x)=k_{1}^{L}+\frac{\epsilon}{1+e^{-\gamma(x-L / 2)}},
$$

where $\epsilon \equiv k_{1}^{R}-k_{1}^{L}$ is the height of the jump. From numerical point of view, we can basically set $k_{1}(0)=k_{1}^{L}$ and $k_{1}(L)=k_{1}^{R}$ for an appropriate system size $L$ with Neumann boundary condition. The parameter $\gamma$ controls the steepness of the slope of $k_{1}$ around the point $x=L / 2$. If the parameter $\gamma$ ap-

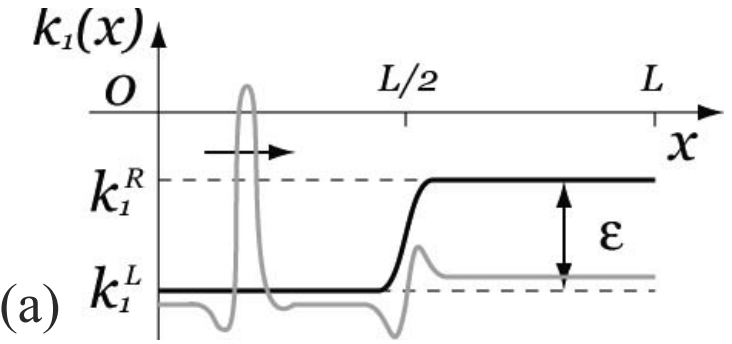

(b)

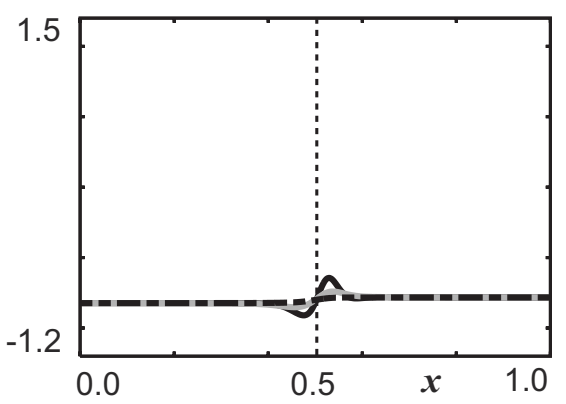

(c)

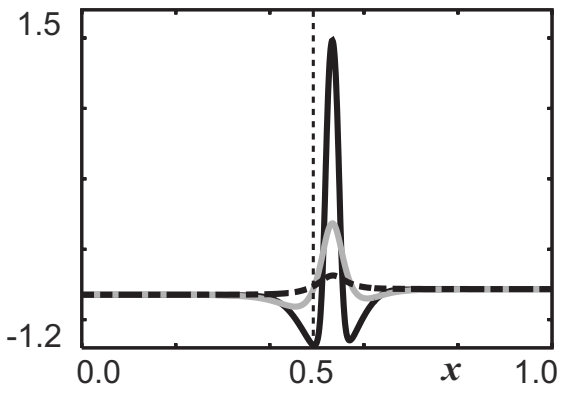

FIG. 5. (a) Schematic picture of initial conditions for heterogeneous case. Solid line shows how $k_{1}(x)$ changes in spatial direction, and gray line displays a manner how a traveling pulse hits small stable defect from left side. The profiles of small and large defect for $\left(k_{1}^{L}, k_{1}^{R}\right)=(-6.75,-6.35)$ are depicted in (b) and (c). The solid, gray and dotted lines indicate the $u, v$, and $w$ component, respectively.

proaches $+\infty, k_{1}(x)$ becomes a step function. In this paper we only consider a steep case (close to a jump discontinuity) with employing $\gamma=100$ hereafter. The pair of parameters $\left(k_{1}^{L}, k_{1}^{R}\right)$ [or, equivalently, $\left.\left(k_{1}^{L}, \epsilon\right)\right]$ is used to specify our heterogeneous system, all the other parameters are fixed as mentioned in Sec. II. We use the Crank-Nicolson method to solve the model (1) with system size $L=4.0$, grid size $\delta x=1.0 \times 10^{-3}, \delta t=0.02$, and $\tau=40.0$ unless otherwise stated.

\section{A. Heterogeneity-induced defects}

The constant background state $U_{0}$ is no more a rest state after introducing the heterogeneity (2) as in Fig. 5(a), since the critical points associated with $k_{1}^{L}$ and $k_{1}^{R}$ are different. The existence of smooth stationary states connecting two critical points (i.e., heteroclinic orbits) is not a trivial question, in fact there are multiple solutions and their stability properties depend on $k_{1}^{L}, \epsilon$, and $\tau$ as will be discussed in subsequent sections. Solving Eq. (1) with appropriate initial data, we found that there are two types of heteroclinic orbits for $\left(k_{1}^{L}, k_{1}^{R}\right)=(-6.75,-6.35)$ called defects: small and large ones 
as shown in Figs. 5(b) and 5(c), which are both locally stable.

In this paper we investigate the dynamics when the traveling pulse encounters a small defect in the parameter space in which both $k_{1}^{L}$ and $k_{1}^{R}$ belong to the interval of $[-6.75,-6.35]$, where stable traveling pulses and stable small defects coexist. It is not always the case that two types of stable defects exist in the above parameter regime, in fact, as we will see in Sec. VI, the large defect does not exist for smaller $k_{L}$ when $\epsilon$ is small.

\section{B. Phase diagram for collision dynamics of traveling pulses with small defects}

In order to make a collision experiment, we first construct a small defect around the jump point, then put a stable traveling pulse far left from the location of the small defect as schematically shown in Fig. 5. Since both traveling pulse and small defect decay exponentially, they do not interfere when the system size $L$ is reasonably large. The outputs of collisions are classified as in the phase diagram of Fig. 6(d) depending on two parameters $\left(k_{1}^{L}, k_{1}^{R}\right)$. The broken line in the middle stands for the homogeneous case where $k_{1}^{L}=k_{1}^{R}$. The upper area of it is the jump-up case of $\epsilon>0$ and the lower part is the jump-down case of $\epsilon<0$. Recall that the pulse is heading to the good environment for $\epsilon>0$ in the sense that traveling velocity is larger in the right region. There are five different outputs: annihilation [Fig. 6(a)], rebound [Figs. 6(b) and 6(e)], penetration [Fig. 6(c)], oscillatory pulse [Fig. 6(f)] and stationary pulse [Fig. 6(g)], depending on the set of $\left(k_{1}^{L}, k_{1}^{R}\right)$.

As is expected, penetration occurs when the difference $|\epsilon|$ is small, i.e., pulses can go across the defect. As $|\epsilon|$ is increased, pulses rebound around the defect point. As $|\epsilon|$ is still increased, the behavior of traveling pulse depends on the sign of $\epsilon$. For the jump-down case $(\epsilon<0)$, the pulse is trapped at the defect point and becomes an oscillatory pulse. For further negative $\epsilon$, the pulse eventually settles down to a stationary state after it hits the defect. On the other hand, for the jump-up case $(\epsilon>0)$, the rebound regime is changed to the annihilation, which is slightly counterintuitive, because the propagating velocity increases when the $k_{1}^{R}$ is increased. Taking a closer look at the behaviors near the boundary of annihilation regime as shown in Fig. 7, we see that the traveling pulse starts to disappear or bounce off just before it touches the left edge of the small defect. A similar behavior is observed in the collision process between two traveling pulses, which typically occurs when the associated parameters are close to a codim 3 singularity consisting of drift, Hopf, and saddle-node bifurcations as discussed in Ref. [26]. In view of Fig. 3(b), our parameter setting falls into the this category and the saddle-node bifurcation is responsible for the occurrence of annihilation, however we do not discuss about it in this paper and delegates this issue to a future work.

\section{COLLISION DYNAMICS IS CONTROLLED BY SCATTORS}

In this subsection, we study the transitions observed in the phase diagram of Fig. 6: first from penetration to rebound, (a)

(e)

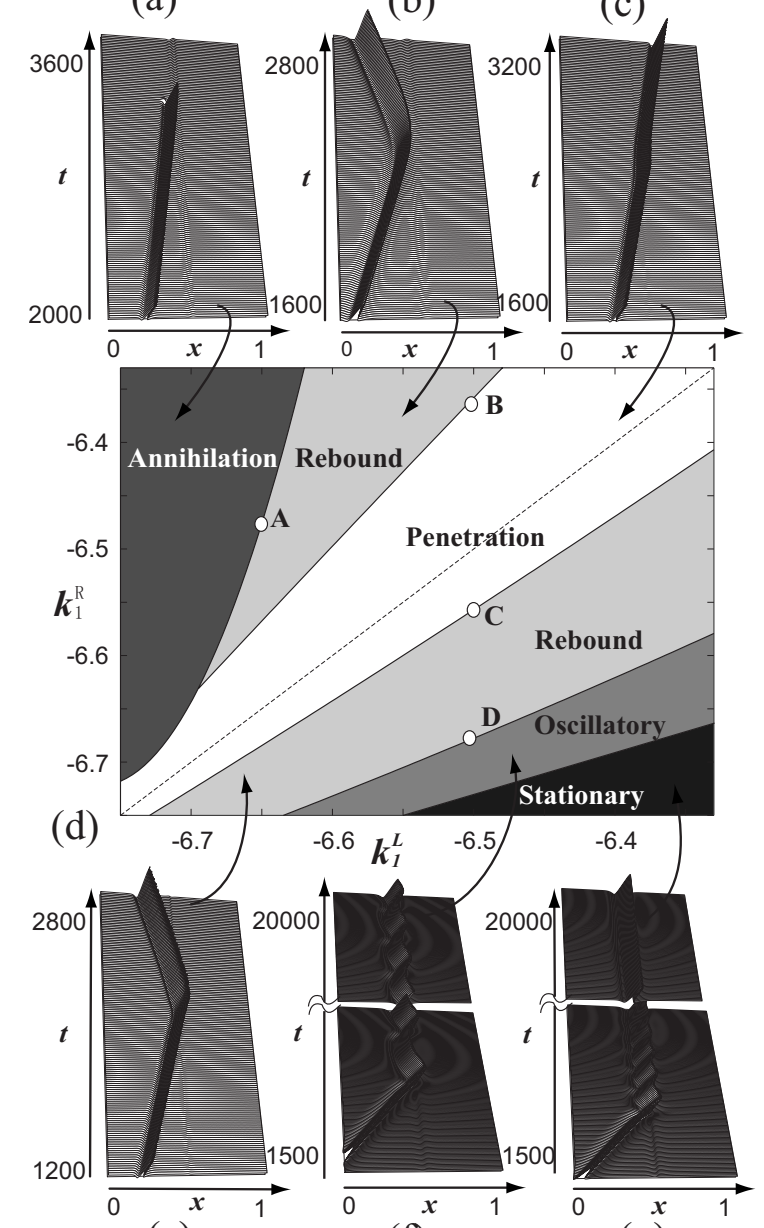

(b)

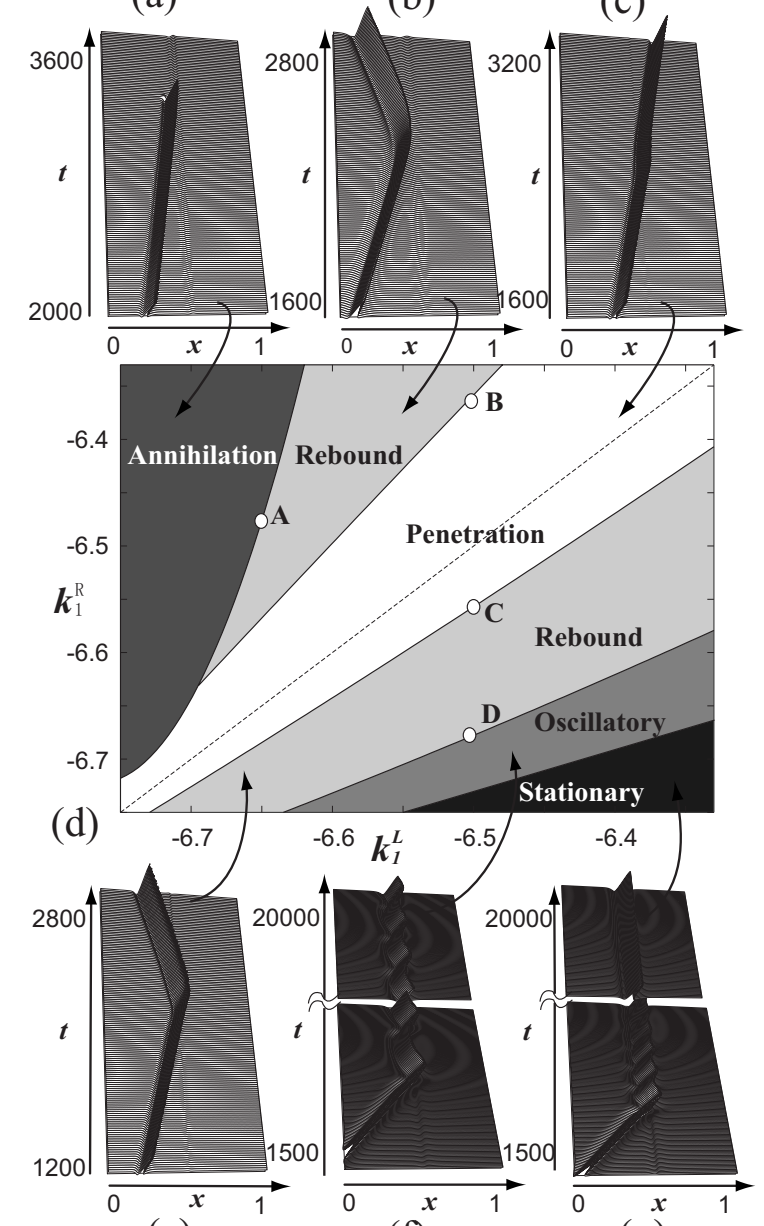

(c)

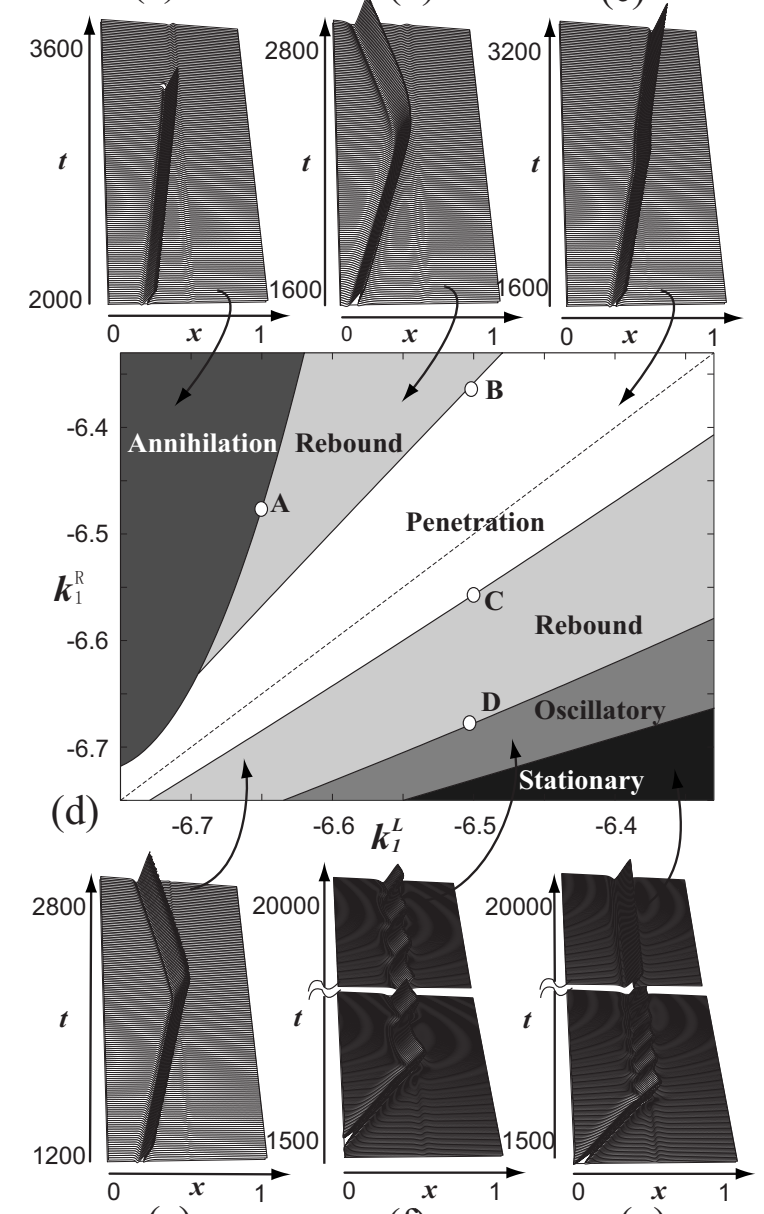

(f)

$(\mathrm{g})$
FIG. 6. The $\left(k_{1}^{L}, k_{1}^{R}\right)$ phase diagram for the responds of traveling pulse hitting small defect pulse. There are five qualitatively different regimes: annihilation (medium gray), rebound (light gray), penetration (white), oscillatory (dark gray), and stationary (black). The details of the pattern dynamics observed at each points marked by white circles are described in the text. Typical spatiotemporal patterns are shown in (a) annihilation for $\left(k_{1}^{L}, k_{1}^{R}\right)=(-6.72,-6.60)$, (b) rebound for $\left(k_{1}^{L}, k_{1}^{R}\right)=(-6.60,-6.40)$, (c) penetration for $\left(k_{1}^{L}, k_{1}^{R}\right)$ $=(-6.60,-6.64)$, (e) rebound for $\left(k_{1}^{L}, k_{1}^{R}\right)=(-6.60,-6.72)$, (f) oscillatory pulse for $\left(k_{1}^{L}, k_{1}^{R}\right)=(-6.50,-6.68)$, and $(\mathrm{g})$ stationary pulse for $\left(k_{1}^{L}, k_{1}^{R}\right)=(-6.40,-6.74)$.

second from stationary to oscillatory, and finally from rebound to oscillatory. It turns out that there appear another type of unstable solutions called scattors which control the behaviors of orbits upon collision with defects in a similar manner discussed in Refs. [15,26]. Also the positions of such scattors clarify the difference of rebound behaviors near the jump point depending on the sign of $\epsilon$.

\section{A. Transition from penetration to rebound}

There are two rebound regions in the phase diagram of Fig. 6(d), however, their behaviors are not exactly the same. For jump-up case of $\epsilon>0$, the pulse bounces before reaching the jump point as shown in Fig. 8(a), while in the jump-down case of $\epsilon<0$, the pulse passes the jump point, then turns 


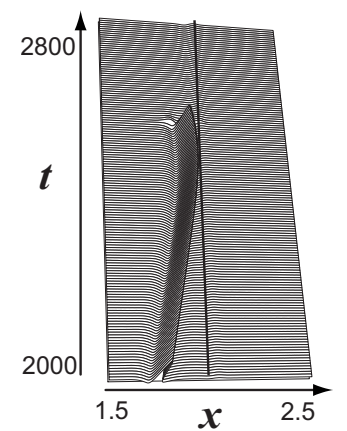

(a)

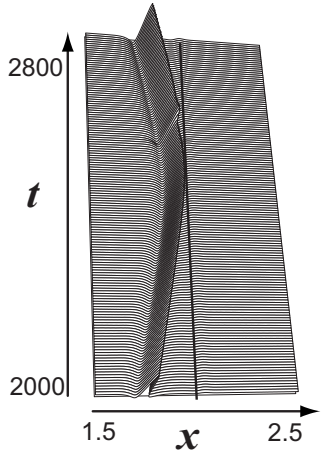

(b)
FIG. 7. Transition from (a) annihilation to (b) rebound occurs around $\left(k_{1}^{L}, k_{1}^{R}\right) \approx(-6.65,-6.486)$ near point A in Fig. $6(\mathrm{~d})$, as $k_{1}^{R}$ is decreased.

back as shown in Fig. 10(a). Two questions arise (i) What is the underlying mechanism for the transition? (ii) How do we explain the difference of rebound manners? To answer these questions, first take a careful look at the behaviors near the transition point $\mathrm{B}$ of $\left(k_{1}^{L}, k_{1}^{R}\right) \approx(-6.50,-6.359)$ in Fig. $6(\mathrm{~d})$. As shown in Fig. 8, the behavior of pulse changes from penetration to rebound when $k_{1}^{R}$ is slightly increased. Quite a long transient is observed around the B point before the pulse makes a decision in which direction to go, i.e., it re-

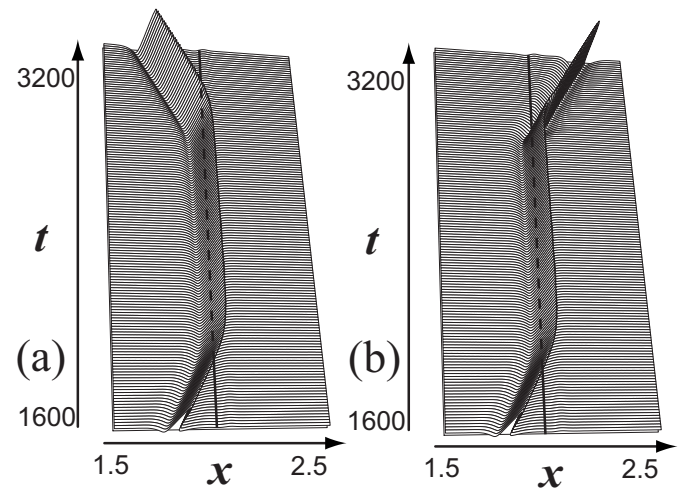

(c)

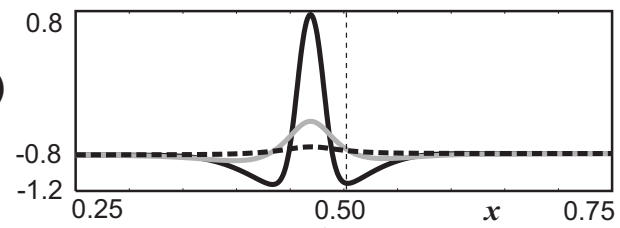

(d)

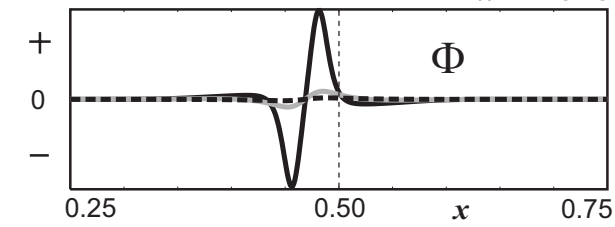

FIG. 8. Transition from rebound to penetration occurs around $\left(k_{1}^{L}, k_{1}^{R}\right) \approx(-6.50,-6.359)$ near point B in Fig. 6(d), as $k_{1}^{R}$ is slightly decreased. Quasisteady pulse is observed near the transition point for both (a) rebound and (b) penetration cases, which is located on the left side of the defect. The profiles of scattor I and its eigenfunction $\Phi$ for $\lambda=0.040836$ are shown in (c) and (d). The solid, gray, and dotted lines indicate the $u, v$, and $w$ component, respectively. mains as a quasisteady state for a certain time after hitting the defect. It is numerically confirmed that, by using the Newton method, there exists a standing pulse, named as scattor I, as depicted in Fig. 8(c), whose peak position is slightly to the left of the jump point, i.e., in the lower side of $k_{1}$ parameter. By solving the linearized eigenvalue problem $\mathcal{L} \Phi=\lambda \Phi$, the scattor I has only one positive real eigenvalue $\lambda=0.040836$. The profile of the associated eigenfunction is shown in Fig. 8(d). The stable manifold of scattor I separates the phase space into two parts and the orbits are sorted out according to which side of the stable manifold it belongs. The destinations of the 1D unstable manifold are penetration and rebound, respectively, which can be confirmed by numerics as shown in Figs. 9(a) and 9(b) by adding a small positive (negative) perturbation respectively to scattor I in the direction parallel to the eigenmode [depicted in Fig. $8(\mathrm{~d})]$. Time evolution of the inner product $\langle W(t, x)$ $\left.-S(x), \Phi^{*}\right\rangle$ in Fig. 9(c) gives us useful quantitative information to predict the fate of orbit, where $W(t, x)$ is a solution of Eq. (1), $S(x)$ is the scattor I, and $\Phi^{*}$ [see Fig. $\left.9(\mathrm{~d})\right]$ is the adjoint eigenfunction of $\lambda$ defined by $\mathcal{L}^{T} \Phi^{*}=\lambda \Phi^{*}$. In fact we see clearly that the inner product changes its sign after collision from positive to negative as $k_{1}^{R}$ is increased, which indicates the transition from penetration to rebound. The duration time how long the orbit stays near the stable manifold of $S(x)$ is a good indicator to detect the transition point. In fact we plot the duration time of the orbit by the criterion $|W(t, x)-S(x)|<0.01$ as a function of $k_{1}^{R}$ with $k_{1}^{L}$ being fixed as -6.50 . The resulting graph is shown in Fig. 9(e) and it has a very sharp high peak at one point. Since $k_{1}^{R}$ is closer to $k_{1}^{R c}$, the longer the duration time, the graph indicates that the transition point locates at $k_{1}^{R c} \approx-6.35946$.

Quite similarly we can find a steady pulse of codim 1 named as scattor II for the transition point $\mathrm{C}$ located around $\left(k_{1}^{L}, k_{1}^{R}\right) \approx(-6.50,-6.557)$. The profiles of scattor II and its associated unstable eigenfunction are shown in Fig. 10. Figure 11 presents the outputs from the scattor II, the inner product with the adjoint eigenfunction, and the plot of the duration time which can be computed in a parallel way to the case of scattor I and it indicates that the transition occurs around $k_{1}^{R} \approx-6.557$ with $k_{1}^{L}$ being fixed as -6.50 . It should be noted, however, that the peak location of scattor II is different from that of scattor I, namely, it sits on the right side of the jump point, i.e., the lower side of $k_{1}$. This explains why the pulse bounces back before (after) the jump point for positive (negative) $\epsilon$.

\section{B. Global relation between defects and scattors}

It is in general not so easy to obtain the unstable objects such as scattors I and II in the previous section. The Newton method can be applied if we have a good candidate of the initial profile for the iteration, however, this does not always work and hence it would be nice if we could have a more systematic method to find such objects. One approach is a continuation method by using path-tracking software such as AUTO [39], which allows us to detect the steady states globally with respect to the parameters. 
(a)

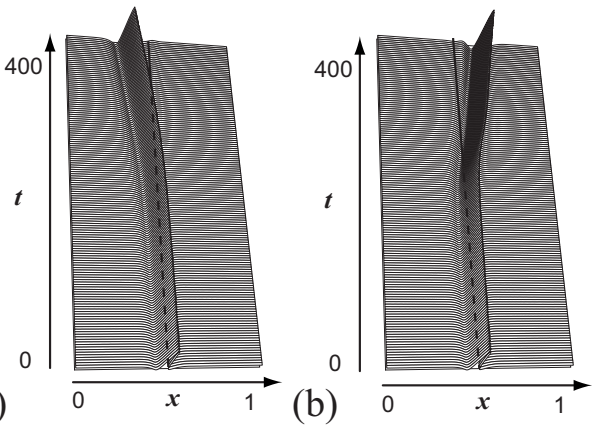

(c)

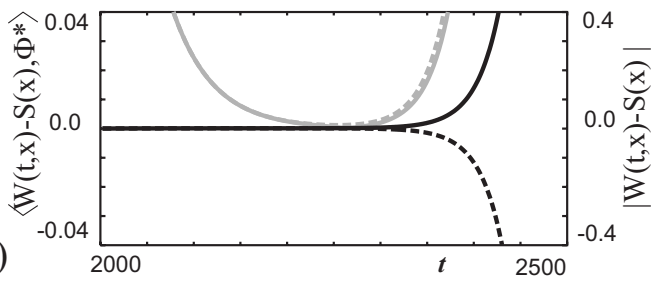

(d)

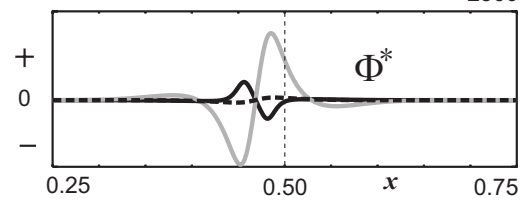

(e)

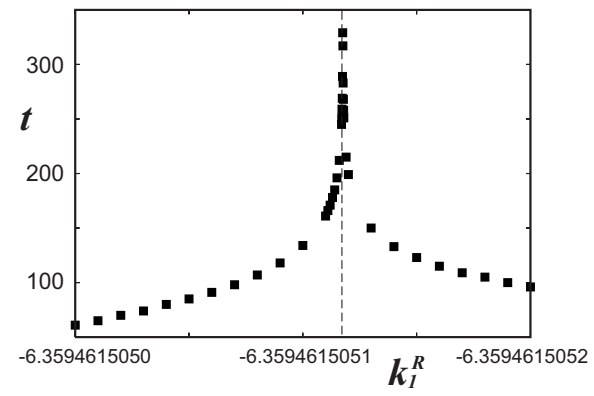

FIG. 9. Outputs from scattor I for $\left(k_{1}^{L}, k_{1}^{R}\right) \approx(-6.50,-6.359)$ of point B in Fig. 6(d). (a) Response of scattor by adding a small negative perturbation in direction of $\Phi$. (b) Response of scattor by adding a small positive perturbation in direction of $\Phi$. (c) Time evolution of the inner product $\left\langle W(t, x)-S(x), \Phi^{*}\right\rangle$ for rebound (penetration) behavior is indicated by the dark (dotted) line. The gray line indicates the associated evolutions of the distance $|W(t, x)-S(x)|$ between the orbit and scattor. (d) The profile of adjoint eigenfunction $\Phi^{*}$, the solid, gray, and dotted lines correspond to the $u, v$, and $w$ component. (e) Time duration when $|W(t, x)-S(x)|<0.01$ for the $k_{1}^{R}$ value.

Starting from a stable large (or small) defect, we can trace the steady pulse solutions with respect to the height of the jump of $\epsilon=k_{1}^{R}-k_{1}^{L}$. Figure 12(a) shows the resulting global bifurcation diagram of steady pulse branches with respect to $\epsilon$ with $k_{1}^{L}$ being fixed as $k_{1}^{L}=-6.50$. The solid lines represent stable solutions and gray lines for unstable ones, in fact these are the continuation of large and small defects discussed in Secs. IV and V. The large stable defect (upper-left branch) decreases its amplitude as $\epsilon$ is increased and is destabilized via the Hopf bifurcation before crossing the another defect branch coming from the right side. The difference between the left and right defect branches is the location of peak, namely, the peak location of the pulse on the left (right) branch sits on the left (right) side of the jump point as is

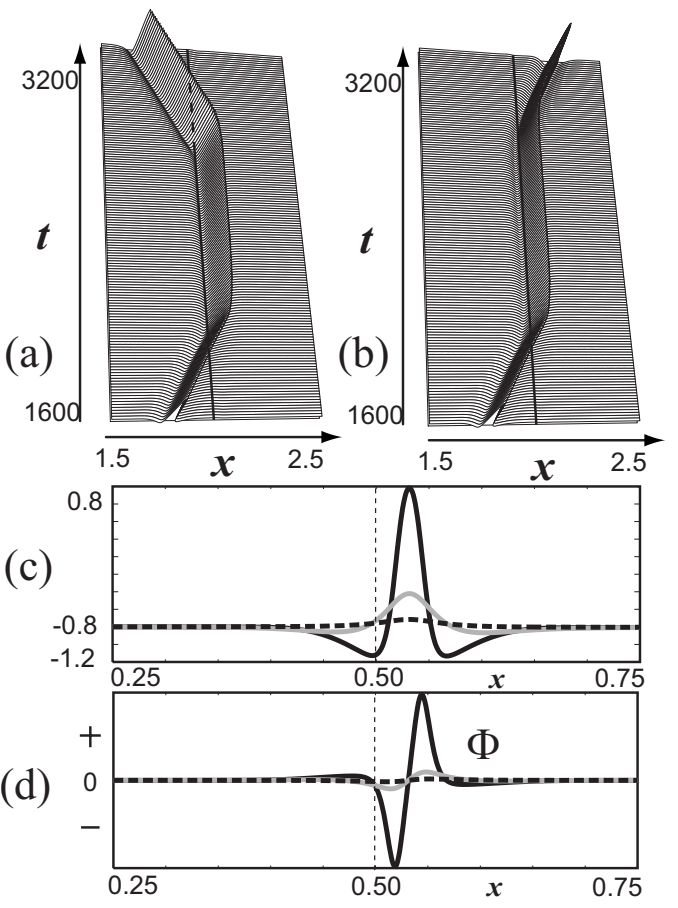

FIG. 10. Transition from rebound to penetration occurs around $\left(k_{1}^{L}, k_{1}^{R}\right) \approx(-6.50,-6.557)$ near point $\mathrm{C}$ in Fig. $6(\mathrm{~d})$, as $k_{1}^{R}$ is slightly increased. Quasisteady pulse is observed near the transition point for both (a) rebound and (b) penetration cases, which is located on the right side of the defect. The profiles of scattor II and its eigenfunction $\Phi$ for $\lambda=0.026259$ are shown in (c) and (d). The solid, gray and dotted lines correspond to the $u, v$, and $w$ components, respectively.

shown in Fig. 12(a) in which the profiles of the $u$ component are depicted along the branch. These branches have two crossing points at $\epsilon=0$ where two types of solutions take exactly the same profiles as those for the homogeneous system in Sec. III. It is conformed numerically that the peak positions of defects move to the center of the domain in the limit of $\epsilon \rightarrow 0$.

The spectral behavior along the upper-left defect branch after the Hopf bifurcation is the following: a pair of complex eigenvalues falls on the real axis and split into two simple real eigenvalues, then one of them approaches to origin as $\epsilon$ is increased and becomes zero translation eigenvalue at $\epsilon$ $=0$. It crosses the origin when $\epsilon$ becomes positive, therefore there is only one positive real eigenvalue around the star point in Fig. 12(a). It turns out that the defect solution of codim 1 at the star point coincides with scattor I of Fig. 8(c). In exactly the same manner, the defect solution of codim 1 located at another star mark coincides with scattor II of Fig. 10(c), therefore scattors are a part of the global defect branches with respect to $\epsilon$.

\section{Transition from stationary to oscillatory pulse}

When the height of the jump becomes negatively large, there appear two regions: oscillatory and stationary ones as shown in Figs. 6(f) and 6(g). The orbit settles down to an ordered pattern after encountering the defect in these regions. 
(a)

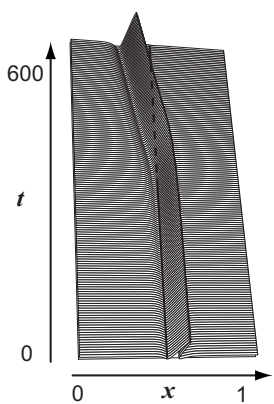

(b)

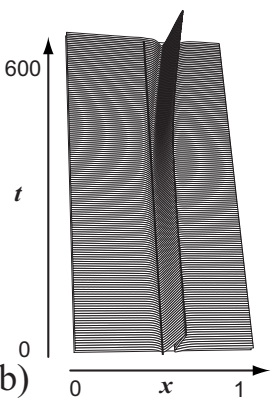

(c)

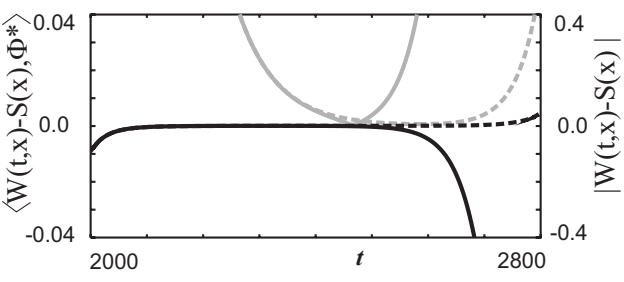

(d)

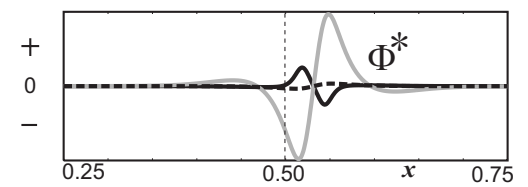

(e)

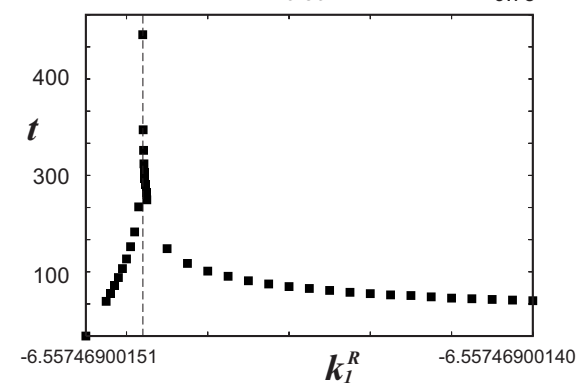

FIG. 11. Outputs from scattor II for $\left(k_{1}^{L}, k_{1}^{R}\right) \approx(-6.50,-6.557)$ of point $\mathrm{C}$ in Fig. 6(d). (a) Response of scattor by adding a small negative perturbation in direction of $\Phi$. (b) Response of scattor by adding a small positive perturbation in direction of $\Phi$. (c) Time evolution of the inner $\left\langle W(t, x)-S(x), \Phi^{*}\right\rangle$ for rebound (penetration) is indicated by the dark (dotted) line. The gray line indicates the associated evolutions of the distance $|W(t, x)-S(x)|$. (d) The profile of adjoint eigenfunction $\Phi^{*}$. The solid, gray and dotted lines corresponds to the $u, v$, and $w$ component, respectively. (e) Time duration when $|W(t, x)-S(x)|<0.01$ for the $k_{1}^{R}$ value.

The evolutional behaviors in each region are depicted in Figs. 13(a) and 13(b). Figure 13(c) plots the position of their pulse peaks. This strongly suggests the emergence of attractors sitting around the jump point. By tracing the defect branch near the Hopf point in Fig. 12(a), such attraction are detected as in the magnified bifurcation diagram of Fig. 13(d). The solid line and filled circles indicate stable steady pulse and stable oscillatory pulses of left-right type emerging via supercritical Hopf bifurcation at the white square in the magnified diagram. The location of the onset of supercritical Hopf bifurcation coincides with that of the transition point from stationary to oscillatory regimes. The boundary between those regions in Fig. 6 is therefore characterized by the Hopf bifurcation line. How about the transition from oscillatory to rebound? The periodic branch has a saddle-node point around $k_{1} \approx-0.17$, which is approximately equal to the transition point from oscillatory to rebound regime. The af-

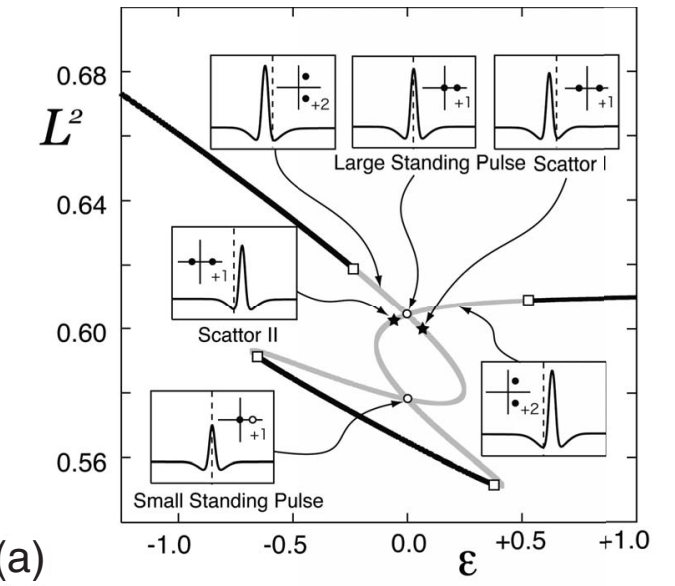

(a)

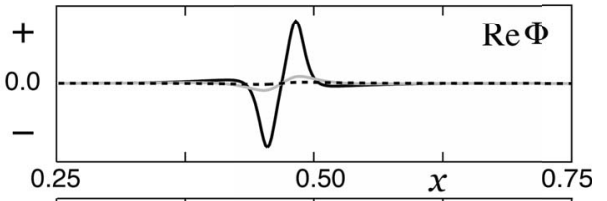

(b)

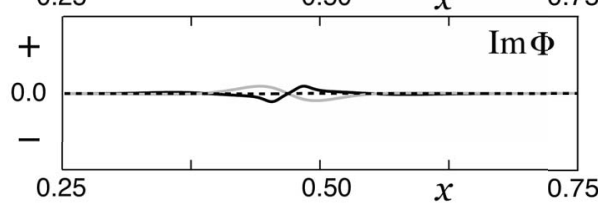

FIG. 12. Global bifurcation diagram for defect pulses for heterogeneous media with $k_{1}^{L}=-6.50$. Solid stars correspond to the codim- 1 scattors observed at the points $\mathrm{B}$ and $\mathrm{C}$, where $\epsilon \approx-0.04$ and 0.13 , respectively. The bifurcation parameter (horizontal axis) is $\epsilon$ with the parameters being fixed the same as in Fig. 3. The profiles of the $u$ component and schematic behaviors of their eigenvalues are depicted along the branch. The locations of pulse peak on the left and right defect branches are different except at $\epsilon=0$. The profiles of the Hopf eigenfunction $\Phi$ of the left-right type for the Hopf point $[\epsilon \approx-0.230$ denoted by the white square in (a)] are depicted in (b). The associated eigenvalue is $\lambda= \pm 0.038 i$. The solid, gray and dotted lines in (b) correspond to the $u, v$, and $w$ component, respectively.

tereffect of the saddle-node point (i.e., temporal oscillation) is, however, not observed even when the parameter is close to the saddle-node point, and a quasisteady state is observed instead as in Fig. 14. This quasisteady state is not captured by the Newton method at present, therefore it is not clear whether the saddle-node point is responsible for the transition or some other basin-switching mechanism should be taken into account.

\section{ORGANIZING CENTER FOR THE HETEROGENEOUS SYSTEM}

One of the main objectives in this paper is to show that traveling pulses, defects, and scattors are the parts of one global branch of an ordered pattern with respect to the parameters $k_{1}, \tau$, and $\epsilon$. They are not separated in a generalized phase space including the parameter space, in fact we already observed in the previous section that scattors can be obtained by continuating the defect branch as $\epsilon$ is varied. This view point leads us to try to find a kind of organizing center which 

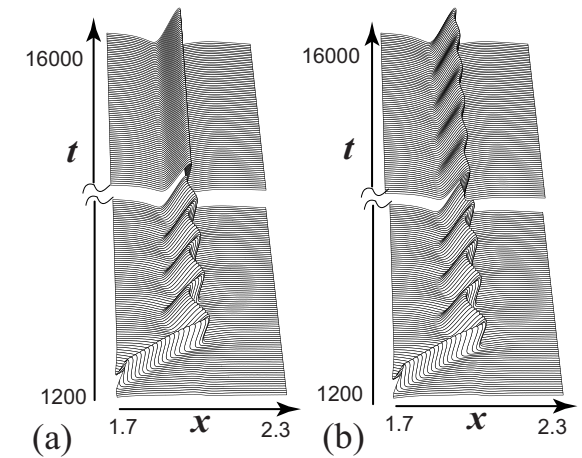

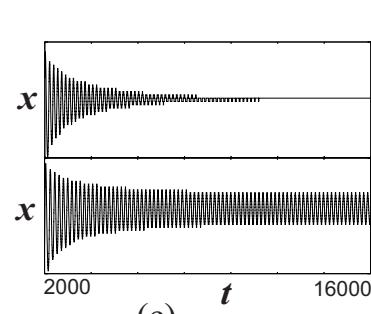

(c)

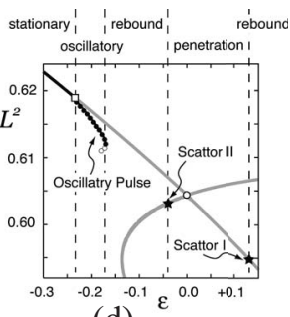

(d)
FIG. 13. Transition occurs from (a) stationary $\left(k_{1}^{R}=-6.74\right)$ to (b) oscillatory $\left(k_{1}^{R}=-6.72\right)$ as $k_{1}^{R}$ is increased with $k_{1}^{L}$ being fixed to be -6.50 . Time evolutions of location of peaks for (a) and (b) are shown in (c). (d) Magnified bifurcation diagram of the upper-left defect branch in Fig. 12(a). The oscillatory defect branch is shown by filled circles.

produces all the above patterns by unfolding appropriate parameters. In this section we present a partial answer to this question.

Recall that standing pulses for homogeneous media can be obtained in the limit of $\epsilon \rightarrow 0$ as in Fig. 12(a). It is interesting to see how this global structure is deformed as $k_{1}^{L}$ is decreased. Figure 15(a) shows the global bifurcation diagram for defect patterns at $k_{1}^{L}=-6.80$. The white squares represent the Hopf bifurcation points. A remarkable thing is that two saddle-node points of left and right defect branches coincide each other at $\epsilon=0.0$ designated by a filled circle. Schematic behaviors of linearized eigenvalues are also depicted along the large defect branch in which the attached numbers show the number of unstable eigenvalues. In view of the upper-left

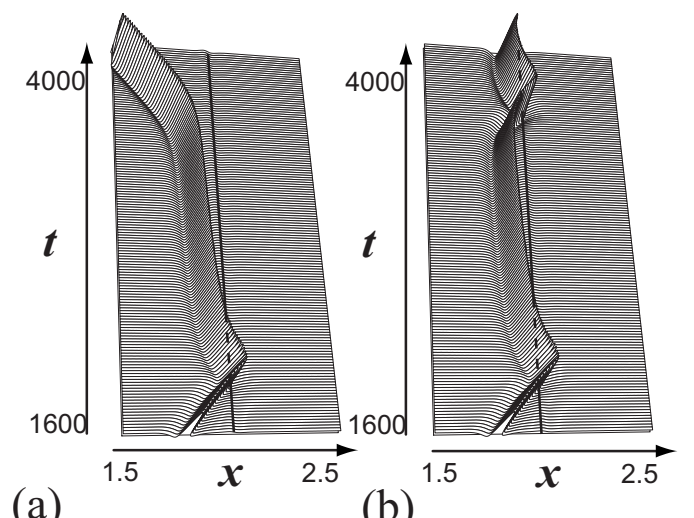

(a)

(b)

FIG. 14. Transition from (a) rebound to (b) oscillatory occurs around $\left(k_{1}^{L}, k_{1}^{R}\right) \approx(-6.50,-6.677)$ near point $\mathrm{D}$ in Fig. $6(\mathrm{~d})$, as $k_{1}^{R}$ is decreased.

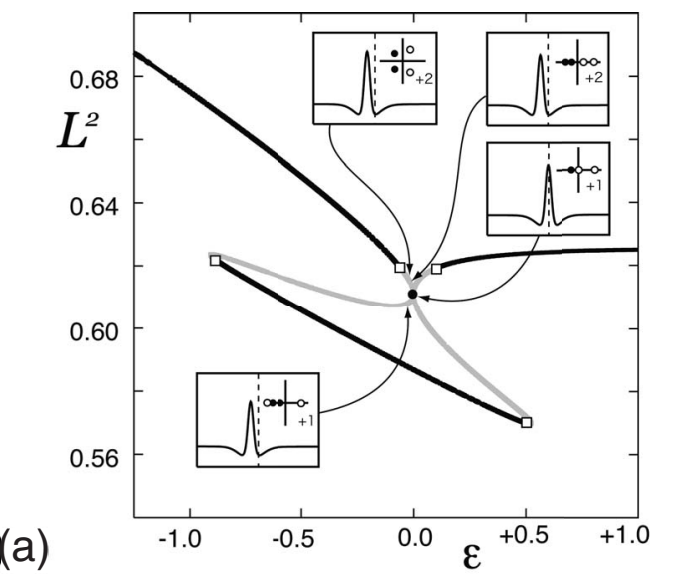

(a)
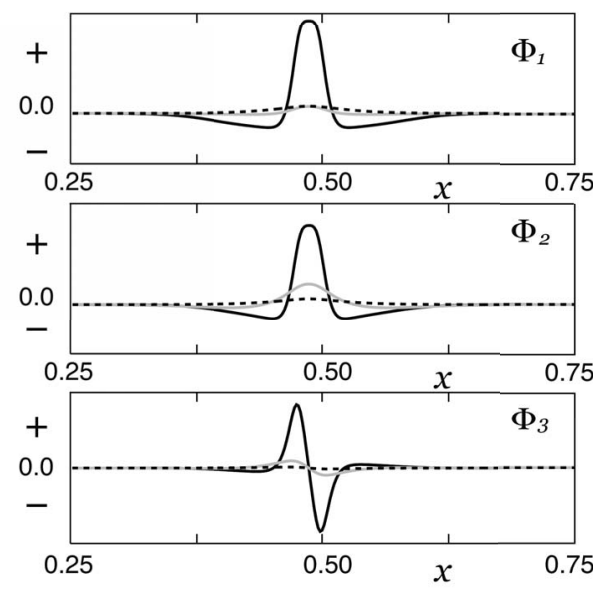

FIG. 15. (a) Global bifurcation diagram for defect pulses for heterogeneous media with $k_{1}^{L}=-6.80$. The profiles of the $u$ component and schematic behaviors of their linearized eigenvalues are depicted along the upper-left branch. Two pairs of complex eigenvalues fall on the real axis and split into four simple real eigenvalues (see upper-right inlet), then one of the real eigenvalues of leftright type (filled circles) with eigenform $\Phi_{3}$ approaches the origin as $\epsilon$ is increased and becomes zero translation eigenvalue at $\epsilon=0$. The two positive real eigenvalues and one negative eigenvalue closer to the origin in the upper-right inlet at $\epsilon=-1.0 \times 10^{-5}$ are given by $\lambda_{1}=0.227, \lambda_{2}=0.007$, and $\lambda_{3}=-0.003$. The profiles of the associated eigenfunctions $\Phi_{1}, \Phi_{2}$, and $\Phi_{3}$ are depicted in (b). Note that $\Phi_{i}(i=1,2)$ inherits the property of symmetry-preserving (updown) motion and $\Phi_{3}$ does that of symmetry-breaking (left-right) one. The solid, gray and dotted lines correspond to the $u, v$, and $w$ component, respectively.

spectral distribution in Fig. 15(a), there are two types of complex eigenvalues with different profiles of their eigenforms: white and filled circles. The white one turns out to have a symmetry-preserving eigenmode (up-down) and the filled one is of symmetry-breaking type (left-right) as in Fig. 15(b). It should be noted that the type of primary Hopf bifurcation is changed from left-right (filled circle) to up-down (white circle) as $k_{1}^{L}$ is decreased from -6.50 to -6.80 as shown in Figs. 12(a), 15(a), and 16(c). These two complex eigenvalues fall onto the real axis as $\epsilon$ becomes closer to zero and each one of them reaches the origin in the homogeneous limit of $\epsilon=0$. More precisely, one of the filled circles (denoted by $\lambda_{3}$ ) becomes the translation zero eigenvalue (sym- 


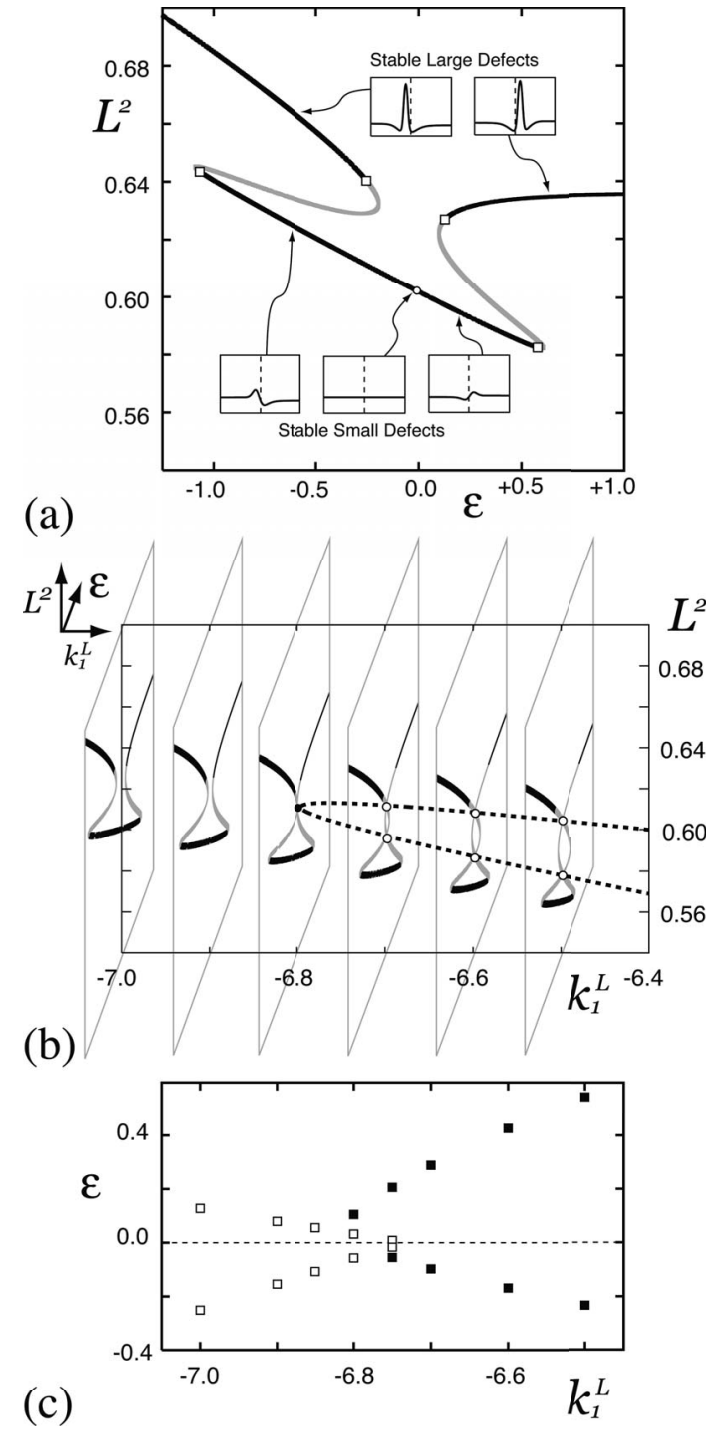

FIG. 16. (a) Global bifurcation diagram for defect pulses in heterogeneous media for $k_{1}^{L}=-7.0$. We use $\epsilon \equiv k_{1}^{R}-k_{1}^{L}$ as the bifurcation parameter. The white squares represent the Hopf bifurcation points. The profiles of the $u$ component are depicted along the branch. Note that the $\epsilon=0$ corresponds to the case of homogeneous media as indicated by the white circle. (b) Global structure of defect branches with respect to $k_{1}^{L}$ and $\epsilon$. The cross section at $\epsilon=0$ (dotted line) coincides with the branch of the standing pulse solutions for homogeneous media in Fig. 3(b). There are two different types of Hopf instabilities on the upper defect branches of Taurus shape depicted in (c): one is left-right type (filled square) and the other is up-down (white square). Each square shows the onset of Hopf instability, which is obtained by projection from upper defect branch to $\left(k_{1}^{L}, \epsilon\right)$ plane. There occurs a crossover around $k_{1}^{L} \approx-6.80$ from left-right type to up-down one as $k_{1}^{L}$ is decreased.

metry breaking) and one of the white circles $\left(\lambda_{2}\right)$ crosses the origin due to the saddle-node bifurcation (symmetry preserving). In fact, as depicted in Fig. 15(b), the eigenfunctions $\Phi_{1}$ and $\Phi_{2}$ suggest an up-down motion, while the $\Phi_{3}$ implies the translation of a left-right direction.

As $k_{1}^{L}$ is still decreased, we have a diagram such as Fig. 16(a) for $k_{1}^{L}=-7.0$. Integrating the diagrams in Figs. 12(c), 15(a), and 16(a), the whole structure of the defect patterns with respect to $k_{1}$ and $\epsilon$ forms a surface similar to the horns of Taurus as shown in Fig. 16(b). It should be noted that the small defect branch (the bottom part of Taurus) is always stable and coincides with the homogeneous constant state at $\epsilon=0$. The branch of standing pulses for the homogeneous media in Fig. 3(b) can be recovered as the cross section at $\epsilon=0$ of the Taurus surface (see the dotted curve). The saddlenode point of the standing pulse branch in Fig. 3(b) is embedded at $\left(k_{1}^{L}, \epsilon\right) \approx(-6.80,0.0)$ denoted by the filled circle. For larger $k_{1}^{L}$ values than -6.80 , the defect pulse surface has two intersecting points with the standing pulse branch for each $k_{1}^{L}$ on the $\epsilon=0$ plane as indicated by the white circles in Fig. 16(b). Recalling that traveling pulses emanate from the standing branch via drift bifurcation and scattors are embedded as solutions in the unstable part of the defect surface, we see that all the relevant solutions can be obtained by continuation starting from the saddle-node point located at $\left(k_{1}^{L}, \epsilon\right) \approx(-6.80,0.0)$. One may call such a point an organizing center for the whole heterogeneity-induced bifurcation including traveling pulses, although two more unfolding parameters in addition to $k_{1}^{L}$ may be necessary to reduce the whole structure to one point of higher singularity.

Finally it should be noted that such a heterogeneityinduced Taurus structure is not only useful to explain the pulse dynamics discussed above, but also gives us insight into various types of complex dynamics created by heterogeneities, for instance, to predict the onset of the pulse generator discussed in the next section (see Fig. 17).

\section{CONCLUSION}

We studied the dynamics of traveling pulses in a heterogeneous media generated by a steplike forcing. The model system studied here is the three-component reaction diffusion system of Eq. (1) of monostable type. Unlike the heterogeneous Gray-Scott model in which heterogeneity is introduced in a multiplicative way (see Ref. [36] for details), the inhomogeneous term $k_{1}(x)$ for Eq. (1) changes the background constant state. This induces a variety of new patterns connecting the left value $k_{1}^{L}$ of the step to the right one $k_{1}^{R}$. We call such heteroclinic solutions as defects. The set of defects forms a Taurus-shaped branch with respect to $k_{1}$ and the height $\epsilon=k_{1}^{R}-k_{1}^{L}$, and it deforms continuously as a function of $k_{1}^{L}$ as presented in Fig. 16. The Taurus basically consists of three parts: a small defect that is a continuation of the constant state for homogeneous case, a large defect that creates traveling pulses via drift bifurcation, and an unstable part connecting small and large defects. These defects are produced by heterogeneities. The theme of this paper is to study the response of a traveling pulse when it encounters a small defect. Note that small defect always exits as a stable stationary state near $\epsilon=0$. The pulse can go across the defect for small $|\epsilon|$, however, it starts to rebound as $|\epsilon|$ become larger as shown in Fig. 6. A remarkable thing is that the unstable part of the defect branch plays the role of separators at the boundary of penetration and rebound regions, namely, there exists a defect of codim 1 at each point of the boundary and the behavior of the orbit is determined by which side of 
(a)

(b)
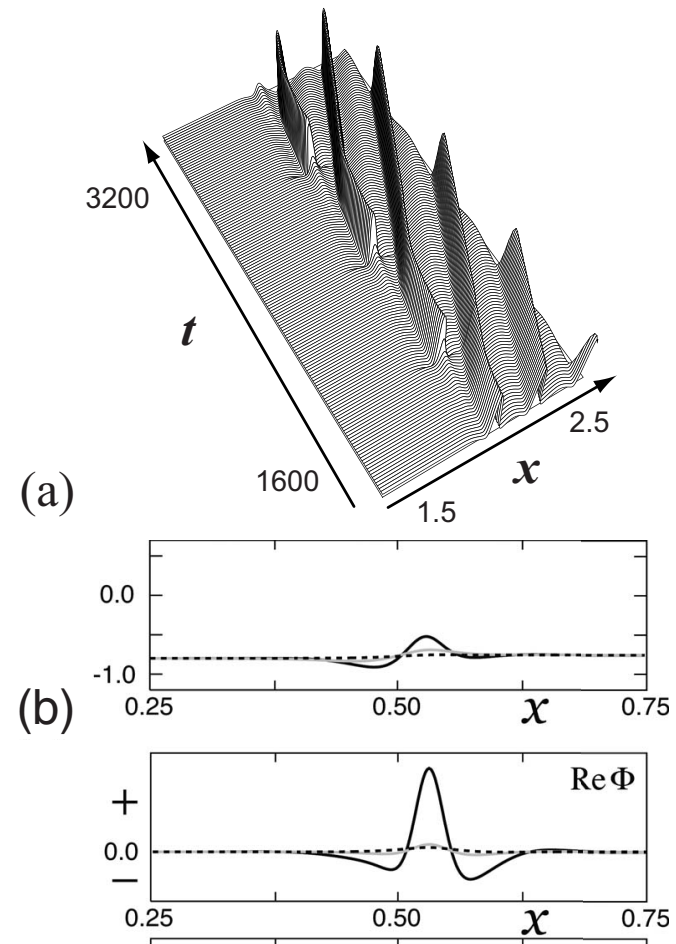

(c)

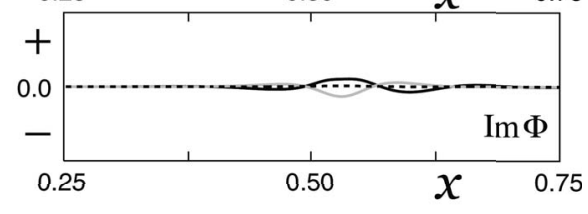

FIG. 17. (a) Spatiotemporal patterns of pulse generator when $k_{1}^{L}=-6.5, k_{1}^{R}=-6.1$, and $\tau=50.0$. The profiles of the unstable small defect pulse and its up-down type eigenfunction are depicted in (b) and (c), respectively. The corresponding eigenvalue is $\lambda$ $=0.013 \pm 0.100 i$. The solid, gray, and dotted lines in (b) and (c) correspond to the $u, v$, and $w$ component, respectively.

the stable manifold of the defect it passes. This is exactly the same type of structure we found for the collision process among pulses and spots $[14,15,40]$. The unstable objects called scattors play the same role as the above defects. This may not be a surprising thing, because propagation in heterogeneous media can be regarded as a collision process between traveling pulse and defect. It should be noted that the viewpoint of unstable objects such as scattors is also useful to understand a different type of complex dynamics such as chaotic transitions discussed in Ref. [41]. When $\epsilon$ is varied in a negative direction (the lower-right part of Fig. 6), the large defect pulse recovers its stability via Hopf bifurcation [see Fig. 13(d)], and therefore the traveling pulses are trapped by either stable large defect or oscillatory defect. On the other hand, when $\epsilon$ is increased (the upper-left part of Fig. 6), annihilation regime appears. In view of the fact that $k_{1}^{L}$ becomes closer to the codim three-singularity consisting of drift, Hopf, and saddle-node bifurcations on the branch of standing pulses for the homogeneous media as in Fig. 3, it is plausible that a similar mechanism discussed in Ref. [26] causes annihilation. The detailed mechanism of this annihilation process is rather complicated, so we will discuss it elsewhere.

The parameter $\tau$ has been fixed to be 40.0 in this paper, however, when $\tau$ becomes larger, we observe various types of complicated behaviors. In fact, for $k_{1}^{L}=-6.50, \epsilon=0.40$, and $\tau=50.0$, in which there are no stable defects, the pulse generator is observed as in Fig. 17 near the inverted Hopf bifurcation of small defect. The small defect oscillates up and down and simultaneously it emits traveling pulses periodically. In view of the global structure of defect branch, the onset of such pulse generator is closely related to the disappearance of stable defects, depending on the positional relation of two Hopf points on small and large defect branches. Pulse generator for Gaussian type heterogeneity was found in Ref. [37], which emits pulses on both sides. From mathematical view point, this generator can be regarded as a heteroclinic orbit connecting unstable oscillation of small defect and wave train of large defects, which may fall in a category discussed in Ref. [42]. Based on the results obtained here, it seems possible to extend our approach to the spatially localized heterogeneities such as bump or Gaussian distributions. For more general heterogeneity like spatial periodic or random case, it would be desirable to reduce PDE dynamics to ODE ones as was done in Ref. [36], in which the effect of heterogeneity is expected to appear in an integral form. We will discuss more about it in a forthcoming paper.

\section{ACKNOWLEDGMENTS}

The authors of this paper would like to thank Dr. K.-I. Ueda for fruitful discussions. This work was partially supported by the Grant-in-Aid for Scientific Research (A)(2) 16204008 and for Young Scientists (B) 18740238 from the Japan Society for the Promotion of Science.
[1] Y. A. Astrov and H.-G. Purwins, Phys. Lett. A 283, 349 (2001).

[2] M. Bär, M. Eiswirth, H.-H. Rotermund, and G. Ertl, Phys. Rev. Lett. 69, 945 (1992).

[3] M. Bode, A. W. Liehr, C. P. Schenk, and H.-G. Purwins, Physica D 161, 45 (2002).

[4] P. de Kepper, J.-J. Perraud, B. Rudovics, and E. Dulos, Int. J. Bifurcation Chaos Appl. Sci. Eng. 4, 1215 (1994).

[5] K. J. Lee, W. D. McCormick, J. E. Pearson, and H. L. Swin- ney, Nature (London) 369, 215 (1994).

[6] A. von Oertzen, A. S. Mikhailov, H.-H. Rotermund, and G. Ertl, J. Phys. Chem. B 102, 4966 (1998).

[7] C. P. Schenk, M. Or-Guil, M. Bode, and H.-G. Purwins, Phys. Rev. Lett. 78, 3781 (1997).

[8] M. Argentina, P. Coullet, and L. Mahadevan, Phys. Rev. Lett. 79, 2803 (1997).

[9] S.-I. Ei, J. Dyn. Differ. Equ. 14, 85 (2002).

[10] S.-I. Ei, M. Mimura, and M. Nagayama, Physica D 165, 176 
(2002).

[11] Y. Hayase and T. Ohta, Phys. Rev. E 62, 5998 (2000).

[12] K. Krischer and A. Mikhailov, Phys. Rev. Lett. 73, 3165 (1994).

[13] J. H. Merkin, V. Petrov, S. K. Scott, and K. Showalter, Phys. Rev. Lett. 76, 546 (1996).

[14] Y. Nishiura, T. Teramoto, and K.-I. Ueda, Phys. Rev. E 67, 056210 (2003).

[15] Y. Nishiura, T. Teramoto, and K.-I. Ueda, Chaos 13, 962 (2003).

[16] Y. Nishiura, Far-from-equilibrium Dynamics (AMS, Metals Park, OH, 2003).

[17] Y. Nishiura and D. Ueyama, Physica D 150, 137 (2001).

[18] T. Ohta, Physica D 151, 61 (2001).

[19] H. G. Purwins, Y. A. Astrov, and I. Brauer, in Proceedings of the Fifth Experimental Chaos Conference, edited by M. Ding, W. L. Ditto, L. M. Pecora, and M. L. Spano. (World-Scientific, Singapore, 2001), pp. 3-13.

[20] W. N. Reynolds, S. Ponce-Dawson, and J. E. Pearson, Phys. Rev. E 56, 185 (1997).

[21] T. Teramoto, K.-I. Ueda, and Y. Nishiura, Phys. Rev. E 69, 056224 (2004).

[22] M. G. Zimmermann, S. O. Firle, M. A. Natiello, M. Hildebrand, M. Eiswirth, M. Bär, A. K. Bangia, and I. G. Kevrekidis, Physica D 110, 92 (1997).

[23] J. Keener and J. Sneyd, Mathematical Physiology (SpringerVerlag, New York, 1998).

[24] Y. Nishiura and D. Ueyama, Physica D 130, 73 (1999).

[25] B. S. Kerner and V. V. Osipov, Autosolitons: A new Approach to Problems of Self-Organization and Turbulence (Kluwer,
Dordrecht, 1994).

[26] Y. Nishiura, T. Teramoto, and K. I. Ueda (unpublished).

[27] M. Gutman, I. Aviram, and A. Rabinovitch, Phys. Rev. E 69, 016211 (2004)

[28] M. Gutman, I. Aviram, and A. Rabinovitch, Phys. Rev. E 70, 037202 (2004).

[29] J. P. Pauwelussen, J. Math. Biol. 15, 151 (1982).

[30] H. Ikeda and M. Mimura, SIAM J. Appl. Math. 49, 515 (1989).

[31] N. Kinezaki, K. Kawasaki, F. Takasu, and N. Shigesada, Theor Popul. Biol. 64, 291 (2003).

[32] J. P. Pauwelussen, Physica D 4, 67 (1981).

[33] J. Xin, SIAM Rev. 42, 161 (2000).

[34] Y.-X. Li, Physica D 186, 27 (2003).

[35] S. Nasuno, Chaos 13, 1010 (2003).

[36] Y. Nishiura, Y. Oyama, and K.-I. Ueda, Hokkaido Math. J. 36(1), 207 (2007).

[37] A. Prat, Y.-X. Li, and P. Bressloff, Physica D 202, 177 (2005).

[38] T. Sugawara and K. Kaneko, Prog. Theor. Phys. Suppl. 161, 344 (2006).

[39] E. J. Doedel and R. C. Paffenroth, AUTO 2000: Continuation and bifurcation sofware for ordinary differential equations (unpublished).

[40] Y. Nishiura, T. Teramoto, and K.-I. Ueda, Chaos 15, 047509 (2005).

[41] M. U. Kobayashi and T. Mizuguchi, Phys. Rev. E 73, 016212 (2006).

[42] B. Sandstede and A. Scheel, SIAM J. Appl. Dyn. Syst. 3, 1 (2004). 